K.A. van der Hucht, G. Koenigsberger \& P.R.J. Eenens, eds.

\title{
Massive stars shaping the ISM: H I holes and shells in nearby galaxies
}

\author{
Elias Brinks \\ Departamento de Astronomía, Universidad de Guanajuato, México
}

\author{
Fabian Walter \\ Radioastronomisches Institut der Universität Bonn, Germany
}

\begin{abstract}
Neutral hydrogen ( $\mathrm{HI}$ ) is a magnificent tool when studying the structure of the interstellar medium (ISM) as it is relatively easily observable and can be mapped at good spatial and velocity resolution with modern instruments. Moreover, it traces the cool $(\sim 100 \mathrm{~K})$ and warm $(\sim 5000 \mathrm{~K})$ neutral gas which together make up about $60 \%$, or the bulk, of the ISM. The currently accepted picture is that stellar winds and subsequent supernovae are the origin for the clearly defined holes or bubbles within the more or less smooth neutral medium. The $\mathrm{H}$ I can therefore serve indirectly as a tracer of the hot interstellar medium (HIM) left behind after the most massive stars within an OB association have gone off as supernovae. A splendid example is the dwarf galaxy IC 2574 for which we discuss $\mathrm{H}$ I, optical and X-ray observations.
\end{abstract}

\section{Introduction}

Several papers in these proceedings touch upon the structure of the ISM (Dopita, these Proceedings; Oey, these Proceedings). This contribution should be read together with their articles.

The field of research dealing with $\mathrm{HI}$ holes and shells has its origins in the discovery by Heiles $(1979,1984)$ of huge, shell-like structures in the $\mathrm{HI}$ distribution of our Galaxy. Somewhat controversial at first, his early findings were magnificently confirmed in the nearest large-scale spirals M 31 (Brinks \& Bajaja 1986) and M 33 (Deul \& den Hartog 1990).

On the basis of scant evidence, Heiles conjectured that stellar winds and supernova explosions of the most massive stars of $\mathrm{OB}$ associations are responsible for creating these structures. This view has generally been accepted and observational and theoretical papers have been published in support. For recent reviews see, e.g., Brinks (1994), van der Hulst (1996), Brinks \& Walter (1998) for observational papers and Tenorio-Tagle \& Bodenheimer (1988) and Mac Low et al. (1999) for more theoretically oriented reviews. Further corroborating evidence comes from a simple model of holes created by $\mathrm{O}$ and B stars developed by Oey \& Clarke (1997) who successfully predict the observed number distribution of holes in the Small Magellanic Cloud (SMC).

Most efforts have concentrated on large spiral galaxies. It is only recently that attention has turned to dwarf irregular galaxies such as Holmberg II (Puche et al. 1992), the SMC (Staveley-Smith et al. 1997), the LMC (Kim et al. 1997) 
and IC 2574 (Walter \& Brinks 1999). Rather counter-intuitively, holes and shells in dwarf galaxies are more dramatic than in larger systems. In retrospect, this can be understood since dwarfs are slow rotators, generally show solid body rotation, and lack density waves. This implies that once features like shells have formed, they will not be deformed by galactic shear and therefore tend to be long lived. Moreover, the overall gravitational potential of a dwarf is much smaller than in a normal spiral. The same amount of energy input of a star forming region therefore has a more pronounced impact on the overall appearance of the ISM, as first shown by Puche et al. (1992).

\section{Observations of dwarf galaxies}

Fig. 1 shows an example of what the neutral gas in a dwarf galaxy looks like. It is an $\mathrm{H}$ I surface brightness map of IC 2574, a dwarf irregular galaxy which belongs to the M 81 group (assumed distance $3.2 \mathrm{Mpc}$ ). The spatial resolution of our maps is about $6^{\prime \prime}$ (or $\sim 95 \mathrm{pc}$ linear resolution); the velocity resolution is $2.6 \mathrm{~km} \mathrm{~s}^{-1}$. Note that the beamsize is so small that it can hardly be properly reproduced. A total of 48 supershells could be identified in this object. More information on IC 2574 is given by Walter et al. (1998). A paper presenting a full analysis of the $\mathrm{H}$ I holes has been submitted (Walter \& Brinks 1999b).

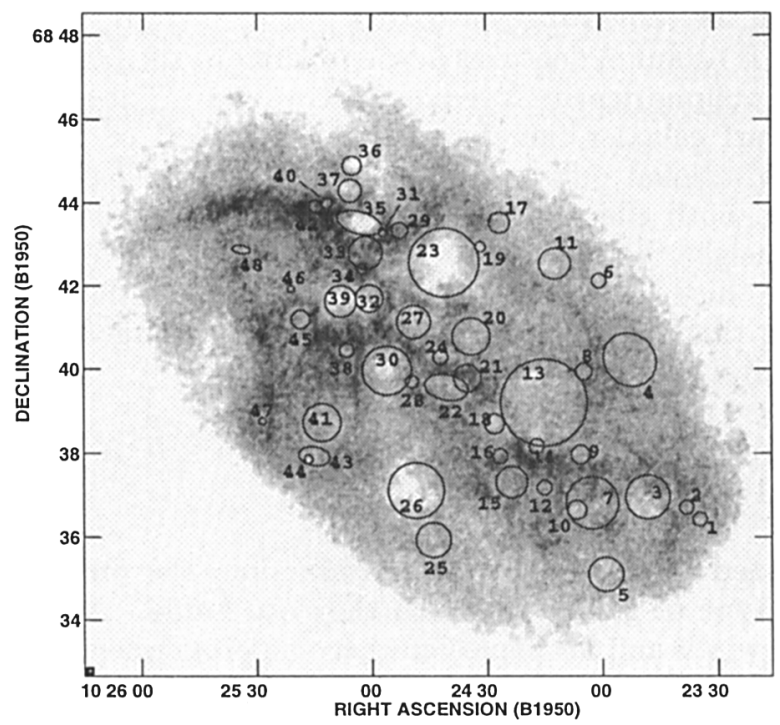

Figure 1. H I surface brightness map of IC 2574. The greyscale is a linear representation of the $H_{I}$ surface brightness. The beam-size $\left(6{ }^{\prime \prime} 4 \times 55^{\prime \prime} 9\right)$ is indicated in the lower left corner. Forty-eight holes were detected whose outlines are indicated by ellipses. The data were obtained with the NRAO-VLA. The NRAO is operated by Associated Universities, Inc., under cooperative agreement with the National Science Foundation. 
In addition to the map shown here and the objects mentioned in the Introduction, several more dwarf galaxies have been observed. We are working on data on DDO 47 (Walter \& Brinks 1999a), NGC 3077 and Holmberg I. Van Dyk et al. (1999) have recently submitted a paper on Sextans A. Hunter, Wilcots and collaborators have data on three more galaxies, IC 10, IC 1613 and NGC 4449 (Hunter et al. 1998), the first object being completely dominated by $\mathrm{H}$ i holes and shells, much like IC 2574 and Ho II. Although none of these objects is classified as a Wolf-Rayet galaxy, many have WR stars located close to or coinciding with regions of active star formacion, such as in IC 10, IC 1613, and IC 2574.

\section{Scale-height and shells}

As was first noticed by Puche et al. (1992) in HoII, the scale-height of the H I layer in dwarf galaxies is larger than in big spiral galaxies. This holds true not only in relative terms, but in absolute terms as well! The aspect ratio of dwarf galaxies tends to be of the order of 10:1 rather than 100:1. Let $h$ be the $1 \sigma$ scale height of a presumed Gaussian $\mathrm{H}_{\mathrm{I}}$ distribution perpendicular to the disk. We find for Ho II, $h=625 \mathrm{pc}$ (Puche et al. 1992); for NGC 5023, $h=460 \mathrm{pc}$ (Bottema et al. 1986); for IC 2574, $h=350 \mathrm{pc}$ (Walter \& Brinks 1999a); and for DDO 47, $h \simeq 700 \mathrm{pc}$, the largest value derived thus far (Walter \& Brinks 1998c). This at first sight somewhat surprising result, can be understood as a direct consequence of the fact that the gravitational potential of a dwarf galaxy is lower than that which is found in spiral galaxies, whereas the measured velocity dispersion of the gas is within the same range $\left(6-9 \mathrm{~km} \mathrm{~s}^{-1}\right)$.

For similar $\mathrm{H}$ I column densities perpendicular to the plane, the above result implies that the volume density is reduced accordingly. The smaller gravitational potential in dwarf galaxies therefore helps the growth of $\mathrm{HI}$ shells in various ways. The gravitational pull on an expanding shell is lower and the ambient density is lower, both effects favouring large shells. Moreover, because of the increased scaleheight, holes have to grow to yet larger dimensions until they break out of the thick $\mathrm{H}$ I layer. This all helps to explain the somewhat counterintuitive finding that the shells in dwarf galaxies are larger, in absolute terms, than in large spiral galaxies.

\section{Statistical properties}

As we alluded to already in the introduction, the properties of $\mathrm{H}$ i holes depend on the type of galaxy in which they are found. As we will show, the effects of stellar winds and SN explosions become progressively more pronounced as a function of Hubble type. In what follows we will compare the observed and derived H I hole properties of IC 2574 with those found in M 31 (Brinks \& Bajaja 1986), an example of a massive spiral galaxy similar to our own; M 33 (Deul \& den Hartog 1990), a less massive spiral; and Ho II (Puche et al. 1992), another dwarf galaxy in the same group of galaxies, yet four times less massive than IC 2574 (Walter \& Brinks 1999a). In other words, the sequence M 31 - M 33 - IC 2574 - HoII spans a large range of different Hubble types from massive spirals to low-mass dwarfs (see Table 1). 
Table 1. Summary of H I hole statistics in four nearby galaxies.

\begin{tabular}{|c|c|c|c|c|}
\hline property & $\mathrm{M} 31^{a}$ & $\mathrm{M} 33^{b}$ & IC $2574^{c}$ & $\mathrm{HoII}{ }^{d}$ \\
\hline linear resolution $(\mathrm{pc})$ & 100 & 55 & 95 & 65 \\
\hline velocity resolution $\left(\mathrm{km} \mathrm{s}^{-1}\right)$ & 8.2 & 8.2 & 2.6 & 2.6 \\
\hline average surface density $\left(10^{20} \mathrm{~cm}^{-2}\right)$ & 5 & 9 & 4 & 10 \\
\hline average volume density $\left(\mathrm{cm}^{-3}\right)$ & 0.6 & 0.45 & 0.15 & 0.2 \\
\hline average velocity dispersion $\left(\mathrm{km} \mathrm{s}^{-1}\right)$ & 8 & 8 & 7 & 7 \\
\hline derived scaleheight $(\mathrm{pc})$ & 120 & 100 & 350 & 625 \\
\hline number of holes & 141 & 148 & 48 & 51 \\
\hline sensitivity per channel $\left(10^{20} \mathrm{~cm}^{-2}\right)$ & 0.3 & 1.0 & 0.5 & 2.4 \\
\hline
\end{tabular}

${ }^{a}$ Brinks \& Bajaja $1986,{ }^{b}$ Deul \& den Hartog 1990, ${ }^{c}$ Walter \& Brinks 1999a, ${ }^{d}$ Puche et al. 1992.

In Fig. 2 and Fig. 3 we compare the distributions of the energies and diameters of the holes in the four galaxies. In both graphs we plot in the form of histograms the relative number of holes, in percent, in order to make a direct comparison possible. To improve the presentation the respective bins for the different objects were slightly shifted when plotting the results.

Fig. 2 shows an overlay of the the distribution of the logarithm of the energies needed to create the observed $\mathrm{H}$ I holes. These energies refer to the initial total energy deposited (using the model by Chevalier 1974), not the currently observed kinetic energy in the $\mathrm{H}$ I shell. We find the very important result that the distributions are identical. Although the derived energies should be treated

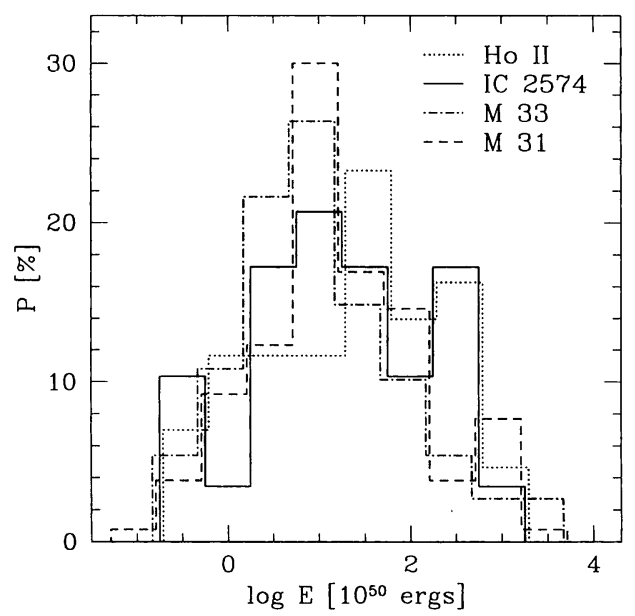

Figure 2. Comparison of the relative distribution, in percentage, of the energies required to produce the $\mathrm{H}$ I holes in IC 2574, M 31, M 33 and Ho II. 


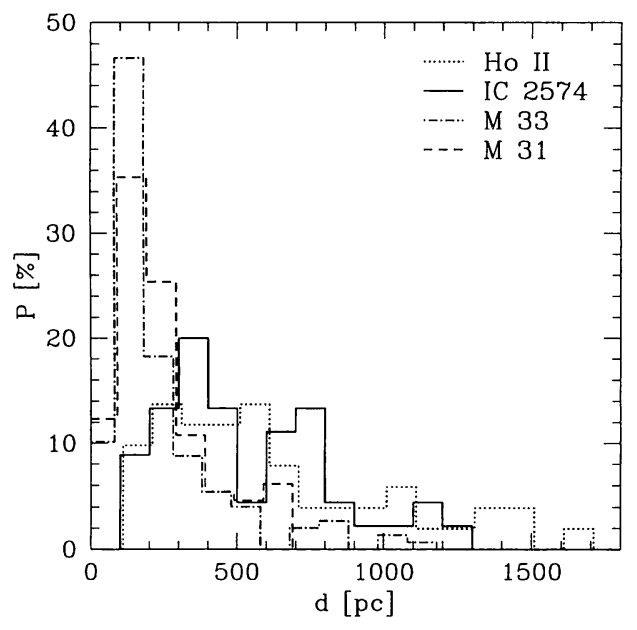

Figure 3. Comparison of the relative distribution, in percentage, of the diameters of the H I holes in IC 2574, M 31, M 33 and Ho II.

as order of magnitude estimates only, it is clear that the global distribution over energy per galaxy is similar. The derived energies range from $10^{50}$ to $10^{53}$ ergs. So, from this we can conclude that regardless of the size, mass and type, the star clusters formed in a typical galaxy are, to first order, identical and deposit more or less the same amount of energy into the ISM.

Fig. 3 shows an overlay of the relative size distribution of the holes found in the four galaxies. In this plot the bins are on a linear scale. Note that there is a clear sequence with Hubble type! The size distribution for holes in M 31 and M 33 cuts off sharply near 600 pc. In contrast, holes in IC 2574 and Ho II reach sizes of 1200 to $1500 \mathrm{pc}$, respectively. The lack of holes with sizes smaller than $\sim 100 \mathrm{pc}$ is due to our resolution limit. As we explained in the previous section, holes are larger for "later" Hubble types because these smaller galaxies have lower masses and hence a lower mass surface density. So, for the same amount of energy deposited, an $\mathrm{H}$ I shell can grow much larger, both because of a lower gravitational potential and a lower ambient density. And because the $\mathrm{H}$ I layer is much thicker as well, shells are prevented from breaking out of the disk.

\section{The case of the supergiant shell in IC 2574}

Obviously, to investigate the sources which created the holes, a multi-wavelength approach is needed. For example, 21-cm observations are required for the identification of the holes as well as for the determination of their kinematics. Optical observations are indispensable to check the stellar distribution and populations within the shells. Narrow band $\mathrm{H} \alpha$ observations are important to trace the regions of current star formation. Quite often, $\mathrm{H} \alpha$-emission is found to be located close to or on the rim of the holes, as defined by the H I-observations. Finally, $\mathrm{X}$-ray observations are important to check whether the cavities of the $\mathrm{H}$ I-holes 


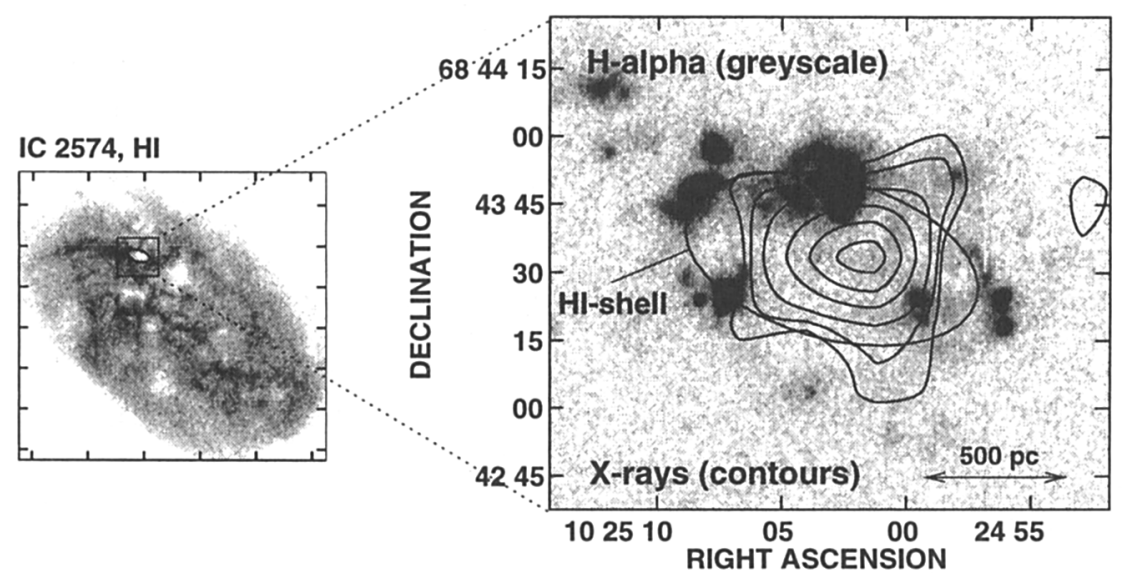

Figure 4. Left: IC 2574 in the $21 \mathrm{~cm}$ line of H I. Right: Blow-up of the supergiant shell in IC 2574. The ellipses plotted in both maps indicate the size of the expanding $\mathrm{H}$ I shell (linear size $\simeq 1000 \times 500 \mathrm{pc}$ ). The greyscale is a representation of the $\mathrm{H} \alpha$ emission coming from the rim of this shell. The contours represent the X-ray emission coming from the inside of the shell as observed with the ROSAT-PSPC camera (for details see Walter et al. 1998). Coordinates are given for B1950.0.

are filled by hot X-ray emitting gas or not. A hot-gas interior is one of the main predictions of theories which state that the holes are created by young OB-associations (see, e.g., Cox \& Smith 1974; Weaver et al. 1977; Chu et al. 1995). To date, only a few shells have been found where such an approach is possible. Examples are the supergiant shell LMC 4 (Bomans, Dennerl \& Kürster 1994), the superbubbles $\mathrm{N} 44$ (Kim et al. 1998) and N 11 (Mac Low et al. 1998), all three situated in the LMC, the supergiant shell SGS 2 in NGC 4449 (Bomans, Chu \& Hopp 1997) and the possible supershell near Holmberg IX (Miller 1995).

The prominent shell in IC 2574 was first seen in high resolution VLA H I observations (Walter \& Brinks 1999a; see also Fig. 4, left hand panel). It has a linear size of about $1000 \mathrm{pc} \times 500 \mathrm{pc}\left(60^{\prime \prime} \times 30^{\prime \prime}\right)$ and is expanding at $\sim 25 \mathrm{~km} \mathrm{~s}^{-1}$. The kinematic age based on the observed size and expansion velocity is estimated at 14 Myr. Narrow-band $\mathrm{H} \alpha$-imaging revealed that current star formation (SF) regions within IC 2574 are predominantly situated on the rim of the $\mathrm{H}$ shell (Fig. 4, right hand panel, greyscale). This suggests that we are witnessing triggered star formation on the rim due to the expansion of the H Ishell (see, e.g., Elmegreen 1994).

A pointed ROSAT observation towards IC 2574 (Walter et al. 1998) revealed that the supergiant shell is indeed filled with extended hot X-ray gas (see the contours in Fig. 4). This makes the supergiant shell in IC 2574 a truly unique region and suggests that we have caught this SGS at an auspicious moment. Assuming a Raymond-Smith (1977) plasma temperature of $\log T=6.8 \pm 0.3 \mathrm{~K}$ and an internal density of $0.03 \pm 0.01 \mathrm{~cm}^{-3}$ we derive an internal pressure of $P \simeq 4 \times 10^{5} \mathrm{~K} \mathrm{~cm}^{-3}$. This pressure is much higher than the pressure of the am- 
bient warm ionized medium ( $P \simeq 10^{3-4} \mathrm{~K} \mathrm{~cm}^{-3}$ ) suggesting that it is probably this hot gas which is still driving the expansion of the shell (see, e.g., Weaver $e t$ al. 1977).

Based on our H I observations and using the models of Chevalier (1974), we derive that the energy required to produce the shell must be of order $10^{53}$ ergs or the equivalent of about 100 Type II SNe. This would mean that the least massive stars that go off as SN are most probably still present in the central stellar association since their lifetimes $(\sim 50 \mathrm{Myr})$ are somewhat longer then the dynamical age of the hole ( $\sim 14 \mathrm{Myr}$, as derived from the $\mathrm{H}$ I observations).

From ground based $R$-band imaging, a giant stellar association is readily visible within the IC 2574-SGS. We speculate that this stellar association is in fact responsible for the formation and expansion of the shell as well as for the heating of the X-ray gas. Unfortunately, the evidence is still largely circumstantial. proposal has been submitted to of this region. Further observations are being planned.

\section{Conclusions}

$\mathrm{H} \mathrm{I}$ observations of sufficient angular and velocity resolution as well as sensitivity of dwarf irregular galaxies are now becoming available. They show that H I shells completely dominate the morphology of dwarf galaxies, such as for IC 2574 or Ho II. We also find that the thickness of the H I layer of dwarf galaxies is substantially larger than in large spiral galaxies.

The supergiant shell in IC 2574 is clearly defined in $\mathrm{H}$ I observations and is surrounded by massive star formation. A pointed ROSAT observation revealed that the cavity enclosed by the supergiant shell is filled with hot, X-ray emitting gas. A prominent stellar association in the center of this SGS is thought to be the powering source for the formation and expansion of the shell as well as for the heating of the interior X-ray emitting gas.

Acknowledgments. The authors are indebted to the Deutsche Forschungsgemeinschaft for financial support in the framework of the Graduiertenkolleg on The Magellanic Clouds and other Dwarf Galaxies. In addition, EB acknowledges a grant awarded by CONACYT (grant number 0460P-E).

\section{References}

Bottema, R., Shostak, G.S., van der Kruit, P.C. 1986, A\&A 167, 34

Brinks, E., Bajaja, E. 1986, A\&A 169, 14

Brinks, E., Walter, F. 1998, in: T. Richtler \& J.M. Braun (eds.), The Magellanic Clouds and Other Dwarf Galaxies, Proc. Bonn/Bochum-Graduiertenkolleg Workshop, p. 1

Bomans, D.J., Dennerl, K., Kürster, M. 1994, A\&A 283, L21

Bomans, D.J., Chu, Y.-H., Hopp, U. 1997, AJ 113, 1678

Brinks, E. 1994, in: G. Tenorio-Tagle (ed.) Violent Star Formation. From 30 Doradus to QSOs (Cambridge: CUP), p. 145

Chevalier, R.A. 1974, ApJ 188, 501

Chu, Y.-H., Chang, H.-W., Su, Y.-L., \& Mac Low, M.-M. 1995, ApJ 450, 156 
Cox, D.P., Smith, B.W. 1974, ApJ 189, L105

Deul, E.R., den Hartog, R.H. 1990, A\&A 229, 362

Elmegreen, B.G. 1994, ApJ 427, 384

Heiles, C. 1979, ApJ 229, 533

Heiles, C. 1984, ApJS 55, 585

Hunter, D.A., Wilcots, E.M., van Woerden, H., Gallagher, J.S., Kohle, S. 1998, ApJ 495, L47

Kim, S., Staveley-Smith, L., Sault, R.J., Kesteven, M.J., McConnell, D., Freeman, K.C. 1997, PASA 14, 119

Kim, S., Chu, Y.-H., Staveley-Smith, L., Smith, R.C. 1998, ApJ 503, 729

Mac Low, M.-M., Klessen, R.S., Burkert, A., Smith, M.D. 1999, Phys. Rev. Let. in press

Mac Low, M.-M., Chang, T.H., Chu, Y.-H., Points, S.D. 1998, ApJ 493, 260

Miller, B.W. 1995, ApJ 446, L75

Oey, M.S., Clarke, C.J. 1997, MNRAS 289, 570

Puche, D., Westpfahl, D., Brinks, E., Roy, J.-R. 1992, AJ 103, 1841

Raymond, J.C., Smith, B.W. 1977, ApJS 35, 419

Staveley-Smith, L., Sault, R.J., Hatzidimitrou, D., Kesteven, M.J., McConnell, D. 1997, MNRAS 289, 225

Tenoric-Tagle, G., Bodenheimer, P. 1988, ARAA 26, 145

van der Hulst, J.M. 1996, in: E.D. Skillman (ed.) The Minnesota Lectures on Extragalactic Neutral Hydrogen, ASP-CS 106, 47

Van Dyk, S.D., Puche, D., Wong, T. 1999, AJ submitted

Walter, F., Brinks, E., Klein, U. 1998, in: T. Richtler \& J.M. Braun (eds.), The Magellanic Clouds and Other Dwarf Galaxies, Proc. Bonn/Bochum-Graduiertenkolleg, p. 299

Walter, F. \& Brinks, E., 1999a, in: The Low Surface Brightness Universe, Proc. IAU Coll. No. 171, in press

Walter, F., Brinks, E., 1999b, AJ submitted

Walter, F., Kerp, J., Duric, N., Brinks, E., Klein, U. 1998, ApJ 502, L143

Weaver, R., McCray, R., Castor, J., Shapiro, P., Moore, R. 1977, ApJ 218, 377

\section{Discussion}

Oey: You stated that disk galaxies with lower H I scale-heights have fewer of the larger shells and lower maximum sizes, and you attributed this to blow-out. Yet the size distributions for M31 and M 33 still show power-law slopes for sizes well above the H I scale-height, thus it seems unclear that blow-out is actually occurring.

E.Brinks: You shouldn't trust observers! Okay, more seriously, there are several possible explanations. Firstly, the largest holes in M 31 and M 33 might be blends (mergers) of smaller (few $100 \mathrm{pc}$ ) holes. The very largest holes in M 33 could be even inter-arm regions (hence artefacts). Lastly, in large galaxies, such as for example M 101, there is evidence that the largest holes could be caused by the infall of a small object, perhaps a dwarf or extragalactic $\mathrm{H}$ I cloud (although this latter mechanism becomes very unlikely in the case of dwarf galaxies).

Diehl: Can you expand your general comments on our Galaxy? I think it would be very interesting to test the $\mathrm{H}$ I hole/hot gas association or nearby $\mathrm{OB}$ associations. We have seen H I shells: (loops I-IV, and recently around Sco-Cen). Here we may have a chance 
for consistency checks (H I , X-rays) in spite of the difficulties, in our galaxy. Is there some 'best' candidate test region?

Brinks: I have always tried to stay away from our Galaxy!. With all due respect for Carl Heiles, who started all this by looking at our Galaxy, the fact that distances are so ambiguous makes detailed correlations between $\mathrm{H}_{\mathrm{I}}$ holes and shells and other components, such as OB associations, tricky.

Gayley: To squeeze more statistics out of your spatial distributions of holes, have you considered applying a 2D-analog of the wavelet analysis, as examplified in the poster by Lépine \& Moffat (these Proceedings)?

Brinks: This might indeed be an interesting approach. However, a more promising technique is that developed by Dave Thilker, René Walterbos and Robert Braun who developed an automated method to search for the signature of expanding shells in nearby galaxies.

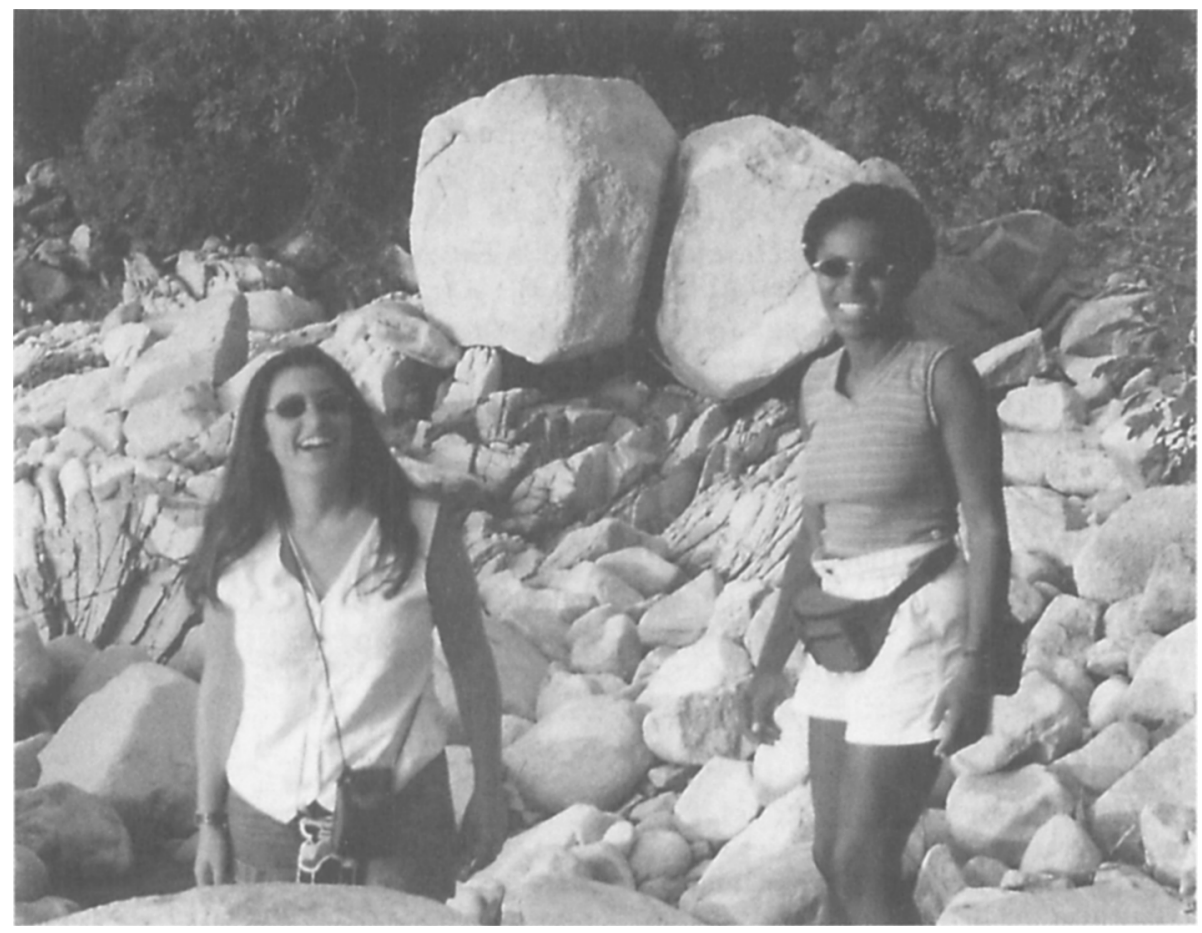

